\title{
Deep-water sediment wave formation: linear stability analysis of coupled flow/bed interaction
}

\author{
L. LESSHAFFT ${ }^{1,2}$, B. HALL ${ }^{2}$, E. MEIBURG ${ }^{2} \dagger$ \\ AND B. KNELLER ${ }^{3}$ \\ ${ }^{1}$ Laboratoire d'Hydrodynamique, CNRS - École Polytechnique, 91128 Palaiseau, France \\ ${ }^{2}$ Department of Mechanical Engineering, University of California, Santa Barbara, CA 93106, USA \\ ${ }^{3}$ Department of Geology and Petroleum Geology, University of Aberdeen, Aberdeen AB24 3FX, UK
}

(Received 31 August 2010; revised 27 February 2011; accepted 7 April 2011;

first published online 18 May 2011)

\begin{abstract}
A linear stability analysis is carried out for the interaction of an erodible sediment bed with a sediment-laden, stratified flow above the bed, such as a turbidity or bottom current. The fluid motion is described by the full, two-dimensional Navier-Stokes equations in the Boussinesq approximation, while erosion is modelled as a diffusive flux of particles from the bed into the fluid. The stability analysis shows the existence of both Tollmien-Schlichting and internal wave modes in the stratified boundary layer. For the internal wave mode, the stratified boundary layer acts as a wave duct, whose height can be determined analytically from the Brunt-Väisälä frequency criterion. Consistent with this criterion, distinct unstable perturbation wavenumber regimes exist for the internal wave mode, which are associated with different numbers of pressure extrema in the wall-normal direction. For representative turbidity current parameters, the analysis predicts unstable wavelengths that are consistent with field observations. As a key condition for instability to occur, the base flow velocity boundary layer needs to be thinner than the corresponding concentration boundary layer. For most of the unstable wavenumber ranges, the phase relations between the sediment bed deformation and the associated wall shear stress and concentration perturbations are such that the sediment waves migrate in the upstream direction, which again is consistent with field observations.
\end{abstract}

Key words: geophysical and geological flows, internal waves, sediment transport

\section{Introduction}

Sediment waves are large-scale, dune-like features on the seafloor, generated through the interaction of a stratified flow with an erodible bed (Wynn et al. 2000b). With typical wavelengths of $O(1-10 \mathrm{~km})$ and heights up to $O(100 \mathrm{~m})$ (Wynn et al. 2000a; Migeon et al. 2001; Nakajima \& Satoh 2001; Normark et al. 2002; Wynn \& Stow 2002), they represent one of the most comprehensively documented bedforms, with the first descriptions dating back to the 1950s (Heezen, Tharp \& Ewing 1959). More recently, a considerable amount of data has been acquired largely as a result of the growth in hydrocarbon exploration in deep-water systems.

The location, structure and orientation of deep-water sediment waves vary, depending on the nature of the flows giving rise to their existence. Wynn \& Stow 
(2002) review and classify the properties of the originating flows, and the character of their bedforms. They identify two types of flows responsible for generating sediment waves: downslope flowing turbidity currents or along-slope flowing bottom currents. Turbidity currents frequently propagate through submarine channels on the continental shelf (Meiburg \& Kneller 2010), and coarse-grained sediment waves can form along the axis of these channels. When a turbidity current encounters a bend in a channel, some of the flow can spill over the levee. This process is known as 'flow stripping' (Piper \& Normark 1983; see review in Peakall, McCaffrey \& Kneller 2000), and the overspill is typically composed of the finer grain sizes initially present in the upper regions of the original turbidity current. The resulting unconfined turbidity current can generate sediment wave fields as it travels down the levee slope, depositing the fine grained sediment (e.g. Normark et al. 1980; Fildani et al. 2006).

Bottom current sediment waves are closely related in nature, and occur on depositional ridges and sediment drifts along the basin floor (Wynn \& Stow 2002). In contrast to turbidity currents, bottom currents are usually not driven by sediment loading, but rather by thermohaline, wind and tidal forces, and they typically attain velocities of 1-20 $\mathrm{cm} \mathrm{s}^{-1}$ (Shanmugam 2006). These flows can persist for thousands of years (Flood 1988), contrasting with the episodic nature of turbidity current flows.

Sediment waves generated by turbidity and bottom currents are generally similar in scale. The crest lines of turbidity current sediment waves are typically aligned transversely to the flow direction, and their dimensions decrease with distance from sediment supply, for example downslope of the levee (Wynn et al. 2000b). Bottom current waves can be oblique or parallel to the flow, depending on how strongly they are influenced by the Coriolis force (Normark et al. 1980). Sediment waves are frequently observed to migrate in the upstream direction (Lonsdale \& Hollister 1979), with downstream migration documented in a few cases (Migeon et al. 2001).

While bottom currents are typically subcritical, turbidity currents may be subor supercritical. Lewis \& Pantin (2002) observed that lee waves may be present in subcritical overbank turbidity current flows, providing a potential link between the two regimes. Normark et al. (1980), Savoye, Piper \& Droz (1993) and Wynn et al. (2000b) pointed out the analogy between sediment waves generated by turbidity currents and fluvial antidunes in supercritical $(F r>1)$ free surface flows (Raudkivi 1966; Kennedy 1969; Reynolds 1976; Engelund \& Fredsoe 1982). Normark et al. (1980) exploited this analogy in order to estimate the flow characteristics responsible for generating sediment waves, by extending the simple two-layer model of Allen (1970) to deep-water currents.

Perhaps the most widely referenced model for deep-sea sediment wave formation is due to Flood (1988). This is based on earlier work by Queney (1948), who derived a closed-form, inviscid velocity field for the internal lee waves developing in stratified flow over sinusoidal topography. Queney's model exhibits a phase shift of $\pi / 2$ between the topography and the tangential velocity component at the surface of the sediment bed. This tangential velocity reaches a maximum on the downstream side of the sediment wave, where the downward slope has its maximum. Hence, Flood (1988) argued that sedimentation should be weakest at this location, and that it might even be possible for erosion to occur. Conversely, the tangential velocity is smallest on the upstream side of the sediment wave, resulting in maximum sedimentation there. In this way, the $\pi / 2$ phase shift results in an effective upstream migration of the sediment wave, consistent with numerous field observations (e.g. Normark et al. 1980). By drawing attention to the importance of internal waves and the phase shift between the sediment waves and the velocity field, Flood's model succeeds at providing a 
qualitative argument for the migration of existing sediment waves. However, it falls short of providing a formal linear stability analysis. The present investigation aims to extend Flood's analysis by providing a quantitative description of the interaction between a stratified flow, internal waves, and an erodible bed via a formal linear stability analysis. Such an analysis is able to capture the details of the underlying instability mechanism and to provide quantitative insight into the roles played by the individual governing dimensionless parameters.

For the case of bedload transport only, a linear stability analysis is provided by Colombini (2004), who demonstrates that a phase shift between the sediment transport and bed topography is required for bedforms to evolve. Colombini \& Stocchino (2008) subsequently extends this work to weakly nonlinear growth of bedforms. The limitation to bedload transport only, and the absence of a density stratification, precludes internal waves from being generated, so that a direct link with the model by Flood (1988) cannot be established.

Nonlinear simulations have also been employed to investigate interactions between turbidity currents and erodible beds (cf. Kubo \& Nakajima 2002). They employ a twodimensional depth-averaged model for a turbidity current flowing over an erodible bed. The simulations show the formation of a regular train of sediment waves after many successive turbidity current deposits, but only if a pre-existing topography is provided. The authors point out that the assumption of an antidune model may not always be appropriate, and they find similarities to the model proposed by Taki \& Parker (2005) and Sun \& Parker (2005) for transportational cyclic steps. Each sediment wave is bounded by hydraulic jumps and the resulting pattern of deposition and erosion causes topographic irregularities to multiply and migrate upstream.

The current investigation addresses the linear stability of deep sea sediment wave formation, within the framework of the full Navier-Stokes equations. In contrast to previous studies that focused on bedload transport, the present analysis aims to elucidate the role of suspended sediment in generating density variations which, in turn, can support the existence of internal waves. The analytical approach corresponds to the one taken by Hall et al. (2008) for investigating the formation of streamwise channels and gullies. While the base flow profiles employed here pertain to turbidity currents, corresponding profiles for bottom currents could easily be substituted. In the following section, the model underlying the analysis is described in some detail, and the relevant dimensionless parameters are identified. Sections 3 and 4 analyse a hierarchy of increasingly complex situations, in order to provide insight into the physical mechanisms underlying the formation of sediment waves. Beginning in $\S 3$ with Tollmien-Schlichting waves in a homogeneous boundary layer, the discussion progresses to constant-density boundary-layer flows over wavy walls, to stratified boundary layers, and in $\S 4$ to flows over erodible beds. In this process, the key instability criterion is derived, and the influence of each dimensionless parameter is analysed in some detail. A summary of the main findings is provided in $\S 5$.

\section{Model formulation}

\subsection{Flow equations}

The model for the interaction of a turbidity current, or correspondingly, a bottom current, with an erodible bed follows the approach introduced by Hall, Meiburg \& Kneller (2008), who described a linear instability mechanism for the formation of submarine channels. The two-dimensional Navier-Stokes equations are written in the Boussinesq approximation, which presumes moderate density differences $(<O(10) \%)$ 


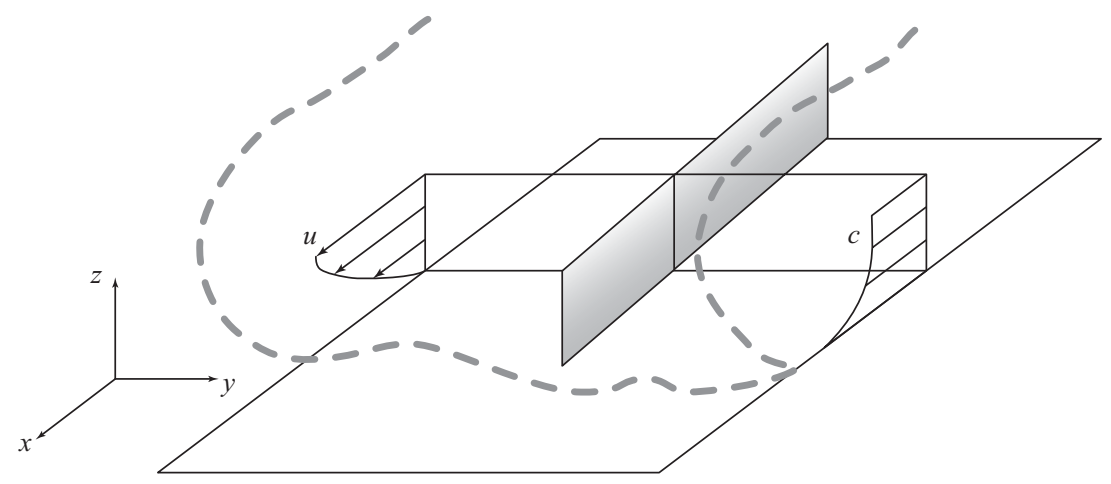

FIGURE 1. Schematic diagram of the flow configuration. The analysis is performed in the fully developed flow region behind the head of a turbidity current in the $(x, z)$-plane.

between particle-laden and clear fluid. The presence of suspended sediment in the fluid is characterized by the volume fraction occupied by the particles, denoted as the concentration field variable $c$. This concentration is assumed to be sufficiently small for fluid-particle interactions to occur predominantly through the momentum equations (2.2)-(2.3), while the effect of particles in the continuity equation (2.1) is taken to be negligible. The analysis considers small, monodisperse sediment particles with negligible inertia and a settling velocity $w_{s}$, whose transport can be modelled by a convection-diffusion equation (2.4). This approach is appropriate for dilute turbidity currents in which the bulk of the sediment is transported in suspension (Necker et al. 2002, 2005), while it would not be adequate for higher density flows where particle-particle interactions and non-Boussinesq effects become important (as in debris flows, for instance). The analysis is performed in the streamwise $x, z$-plane, as defined in figure 1, some distance behind the head of a sustained turbidity current. All flow variables are assumed to be independent of the spanwise coordinate $y$, and the spanwise velocity component $v$ is taken to be zero. The two-dimensional flow field is governed by

$$
\begin{aligned}
\frac{\partial u}{\partial x}+\frac{\partial w}{\partial z} & =0 \\
\frac{\partial u}{\partial t}+u \frac{\partial u}{\partial x}+w \frac{\partial u}{\partial z} & =-\frac{1}{\rho_{f}} \frac{\partial p}{\partial x}+v\left(\frac{\partial^{2} u}{\partial x^{2}}+\frac{\partial^{2} u}{\partial z^{2}}\right) \\
\frac{\partial w}{\partial t}+u \frac{\partial w}{\partial x}+w \frac{\partial w}{\partial z} & =-\frac{1}{\rho_{f}} \frac{\partial p}{\partial z}+v\left(\frac{\partial^{2} w}{\partial x^{2}}+\frac{\partial^{2} w}{\partial z^{2}}\right)-\frac{\rho}{\rho_{f}} g, \\
\frac{\partial c}{\partial t}+u \frac{\partial c}{\partial x}+\left(w-w_{s}\right) \frac{\partial c}{\partial z} & =D\left(\frac{\partial^{2} c}{\partial x^{2}}+\frac{\partial^{2} c}{\partial z^{2}}\right) .
\end{aligned}
$$

The two-dimensional fluid velocity vector has components $u$ and $w$. Since we aim at a conceptual investigation of the instability mechanisms involved in deep-sea sediment wave formation, viscosity and diffusivity are assumed to be constant. Although this choice may be interpreted as the simplest form of the eddy viscosity in a turbulent flow, the effects of turbulence in a real turbidity current would not be appropriately modelled by means of a constant eddy viscosity. However, the precise form of the turbulent stress tensor is unknown in such a complex flow (variable density, high particle concentration near an erodible bed, effects of collective behaviour). Stacey \& 
Bowen (1988), for instance, attempted to determine parameters for a variable-viscosity model in turbidity currents to match experimental data; their study illustrates the difficulty of formulating a model that may be extrapolated to field-scale flow regimes. In the light of such uncertainties, we feel that the simplest assumption of constant eddy viscosity is best suited for an investigation of the salient instability mechanisms.

The monodisperse particles are advected by the fluid, while settling with a constant velocity $w_{s}$. The density $\rho$ of the suspension is linearly related to the volume fraction $c$ of the particles by $\rho(\boldsymbol{x})=\rho_{f}[1+\gamma c(\boldsymbol{x})]$, where $\gamma=\left(\rho_{p}-\rho_{f}\right) / \rho_{f}$ is the excess density ratio based on the fluid density $\rho_{f}$ and the particle material density $\rho_{p}$.

\subsection{Fluid/bed interface evolution}

To analyse the coupled dynamics of the turbidity current and the interface between the fluid and the sediment bed, a model is required that describes how the interface location changes with time as a result of sediment deposition and erosion. Towards this end, the interface position is defined by its elevation $z=\eta(x, t)$ above the $x$-axis. It is assumed that sediment in the turbidity current is predominantly transported as suspended load, due to high wall shear, whereas the effects of bedload are not accounted for in the present model. Following Hall et al. (2008), the interface elevation is taken to evolve according to

$$
\frac{\partial \eta}{\partial t}=w_{s} c(z=\eta)-\beta \frac{\tau_{n}}{n_{z}} .
$$

Here, $\beta$ quantifies the rate at which particle volume is eroded per unit area and unit shear stress, $\tau_{n}$ denotes the shear stress acting at the surface of the sediment bed, and $n_{z}$ is the vertical projection of the wall-normal unit vector. Equations (2.1)-(2.5) comprise the governing equations for the coupled flow/sediment bed dynamics. At the interface, a no-slip condition is imposed for the horizontal $u$-velocity component, while the vertical $w$-velocity component has to equal the rate at which the interface advances or recedes,

$$
u(z=\eta)=0, \quad w(z=\eta)=\frac{\partial \eta}{\partial t} .
$$

The interstitial void fraction in the sediment bed is not taken into account; assuming no spatial variation in packing, a constant packing ratio would multiply the right-hand side of the boundary condition for $w$. The erosive transport of sediment from the bed into the interior of the fluid is modelled as a diffusive flux (Parker 1978; Blanchette et al. 2005), expressed as a boundary condition for the wall-normal concentration gradient,

$$
\left.D \frac{\partial c}{\partial n}\right|_{z=\eta}=-\beta \tau_{n} .
$$

The flow over an unchanging bottom topography will be considered in $\S 3$. In that case, (2.5) is simply replaced by

$$
\frac{\partial \eta}{\partial t}=0 .
$$

\subsection{Non-dimensionalisation and choice of parameters}

The governing equations are rendered dimensionless with respect to a diffusive length scale $l=D / w_{s}$, the outer flow velocity $u_{\infty}$ and the fluid density $\rho_{f}$. The concentration $c$ already represents a non-dimensional volume fraction, and, in contrast to Hall et al. (2008), is not renormalized in the present framework. 
During the formation of sediment waves, there may be an overall net deposition (or erosion) of particles. The following analysis will be carried out in a reference frame that moves with the uniformly advancing (or receding) interface. If the unperturbed interface moves with a dimensionless velocity $\mathrm{d} \eta / \mathrm{d} t=w_{a v}$ in the laboratory reference frame, we introduce $z^{\prime}=z-w_{a v} t, \eta^{\prime}=\eta-w_{a v} t$ and $w^{\prime}=w-w_{a v}$. The NavierStokes equations are invariant under a constant-velocity translation. If the primes are omitted, the non-dimensional equations in the co-moving frame of reference read

$$
\begin{gathered}
\frac{\partial u}{\partial x}+\frac{\partial w}{\partial z}=0 \\
\frac{\partial u}{\partial t}+u \frac{\partial u}{\partial x}+w \frac{\partial u}{\partial z}=-\frac{\partial p}{\partial x}+\frac{1}{R e}\left(\frac{\partial^{2} u}{\partial x^{2}}+\frac{\partial^{2} u}{\partial z^{2}}\right) \\
\frac{\partial w}{\partial t}+u \frac{\partial w}{\partial x}+w \frac{\partial w}{\partial z}=-\frac{\partial p}{\partial z}+\frac{1}{R e}\left(\frac{\partial^{2} w}{\partial x^{2}}+\frac{\partial^{2} w}{\partial z^{2}}\right)-G c \\
\frac{\partial c}{\partial t}+u \frac{\partial c}{\partial x}+\left(w-W_{s}\right) \frac{\partial c}{\partial z}=W_{s}\left(\frac{\partial^{2} c}{\partial x^{2}}+\frac{\partial^{2} c}{\partial z^{2}}\right) \\
\frac{\partial \eta}{\partial t}=-w_{a v}+W_{s} c(z=\eta)-N \frac{\tau_{n}}{n_{z}}
\end{gathered}
$$

with boundary conditions at the interface

$$
\begin{aligned}
u(z=\eta) & =0 \\
w(z=\eta) & =\frac{\partial \eta}{\partial t}, \\
\left.\frac{\partial c}{\partial n}\right|_{z=\eta} & =-\frac{N}{W_{s}} \tau_{n} .
\end{aligned}
$$

The Reynolds number, based on the thickness of the concentration boundary layer, is defined as $R e=u_{\infty} D / \nu w_{s}$. Due to the definition of the length scale, the non-dimensional quantity $W_{s}=w_{s} / u_{\infty}$ plays a dual role as the settling speed and as the dimensionless particle diffusion coefficient, i.e. particle Peclet number. The influence of buoyancy on the flow is governed by a gravity parameter $G=g^{\prime} D / u_{\infty}^{2} w_{s}$, where the reduced gravity is defined as $g^{\prime}=g\left(\rho_{p}-\rho_{f}\right) / \rho_{f}$. The erosion coefficient $N=\beta v \rho_{f} w_{s} / D$ relates the bottom shear stress to the velocity at which the interface recedes as a result of erosion.

Parameter values identical to those used in Hall et al. (2008) are considered as a standard configuration in the present analysis. These values were estimated from experimental literature: Fildani et al. (2006) describe characteristic property values of flow-stripped turbidity currents with $u_{\infty}=5 \mathrm{~m} \mathrm{~s}^{-1}$. The sediment is composed of fine silt/clay particles, which tend to flocculate with an effective settling velocity of the order of $10^{-3} \mathrm{~m} \mathrm{~s}^{-1}$ (Hill 1998). The effective turbulent eddy viscosity and diffusivities are set to $v_{T}=D=0.1 \mathrm{~m}^{2} \mathrm{~s}^{-1}$. This leads to non-dimensional parameter values $R e=5000, W_{s}=0.0002$ and $G=10$. The assumption of a linear relation between the bottom shear stress and the erosion rate represents a first-order approximation of the nonlinear threshold behaviour observed by Garcia \& Parker (1993). From their measurements, we conservatively estimate $N \leqslant O\left(10^{-5}\right)$, and we choose $N=10^{-5}$ as a typical value. The present study therefore pertains to turbidity currents with 
sufficiently strong bottom shear stress, above the threshold required for erosion of sediment.

\subsection{Linear perturbations in a steady parallel base flow}

The flow variables $\boldsymbol{q}=(u, w, p, c, \eta)^{\mathrm{T}}$ are written as a superposition $\boldsymbol{q}=\boldsymbol{q}_{0}+\boldsymbol{q}^{\prime}$, where the perturbation components $\boldsymbol{q}^{\prime}(x, z, t)$ evolve within a steady parallel base flow $\boldsymbol{q}_{0}(z)$. The model base flow around which the equations of motion are linearized is derived in the same manner as in Hall et al. (2008) (see also Stacey \& Bowen 1988), except that here the concentration distribution is not normalized with respect to its value $c_{\infty}$ at $z \rightarrow \infty$. The base flow profiles of streamwise velocity and concentration are thus obtained as

$$
\begin{aligned}
& u_{0}(z)=1-\mathrm{e}^{-z / L}, \\
& c_{0}(z)=\frac{N}{L W_{s}} \mathrm{e}^{-z}+c_{\infty},
\end{aligned}
$$

while $w_{0}(z)$ and $\eta_{0}$ are zero by construction. The freely prescribed parameter $L$ denotes the ratio of velocity boundary-layer thickness to concentration layer thickness. This base flow satisfies the boundary conditions (2.14) and (2.16), although it requires the assumption of a synthetic pressure gradient, as discussed in detail by Hall et al. (2008). The explicit expression for $p_{0}$ is not required for the instability analysis. Based on experimental observations that typically show the velocity boundary layer in a developed turbidity current to be thinner than the concentration layer (see for instance Peakall et al. 2000), $L=0.1$ will be chosen as a standard value throughout this study. The investigation is limited to the boundary layer of a turbidity current in order to minimize the number of free parameters needed to characterize the base flow. Boundary-layer instability modes are concentrated in the flow region near the sediment bed and are largely unaffected by the base flow features outside this region. Conversely, instabilities that exist due to the finite height of a complete turbidity current profile are confined to the outer region, and are therefore expected to interact less strongly with the sediment bed.

The governing equations and boundary conditions (2.9)-(2.16) are linearized about the base flow (2.17) and (2.18), and perturbation quantities are expressed in the form of normal modes

$$
\boldsymbol{q}^{\prime}(x, z, t)=\boldsymbol{Q}(z) \mathrm{e}^{\mathrm{i} \alpha x+\sigma t}+\text { c.c. },
$$

with real streamwise wavenumber $\alpha$, complex temporal growth rate $\sigma$ and complex amplitude functions $\boldsymbol{Q}(z)=[U(z), W(z), P(z), C(z), E]^{\mathrm{T}}$. With these substitutions, the linear perturbation equations become

$$
\begin{aligned}
& \mathrm{i} \alpha U+\frac{\mathrm{d} W}{\mathrm{~d} z}=0 \\
& \sigma U+u_{0} \mathrm{i} \alpha U+W \frac{\mathrm{d} u_{0}}{\mathrm{~d} z}=-\mathrm{i} \alpha P+\frac{1}{R e}\left(-\alpha^{2} U+\frac{\mathrm{d}^{2} U}{\mathrm{~d} z^{2}}\right) \\
& \sigma W+u_{0} \mathrm{i} \alpha W=-\frac{\mathrm{d} P}{\mathrm{~d} z}+\frac{1}{R e}\left(-\alpha^{2} W+\frac{\mathrm{d}^{2} W}{\mathrm{~d} z^{2}}\right)-G C \\
& \sigma C+u_{0} \mathrm{i} \alpha C+W \frac{\mathrm{d} c_{0}}{\mathrm{~d} z}-W_{s} \frac{\mathrm{d} C}{\mathrm{~d} z}=W_{s}\left(-\alpha^{2} C+\frac{\mathrm{d}^{2} C}{\mathrm{~d} z^{2}}\right)
\end{aligned}
$$




$$
\sigma E=\left.E W_{s} \frac{\mathrm{d} c_{0}}{\mathrm{~d} z}\right|_{z=0}-\left.E N \frac{\mathrm{d}^{2} u_{0}}{\mathrm{~d} z^{2}}\right|_{z=0}+W_{s} C(0)-\left.N \frac{\mathrm{d} U}{\mathrm{~d} z}\right|_{z=0},
$$

with boundary conditions

$$
\begin{aligned}
& U(0)+\left.E \frac{\mathrm{d} u_{0}}{\mathrm{~d} z}\right|_{z=0}=0 \\
& W(0)=\sigma E \\
&\left.E \frac{\mathrm{d}^{2} c_{0}}{\mathrm{~d} z^{2}}\right|_{z=0}+\left.\frac{\mathrm{d} C}{\mathrm{~d} z}\right|_{z=0}=-\frac{N}{W_{s}}\left(\left.E \frac{\mathrm{d}^{2} u_{0}}{\mathrm{~d} z^{2}}\right|_{z=0}+\left.\frac{\mathrm{d} U}{\mathrm{~d} z}\right|_{z=0}\right), \\
&\left.\frac{\mathrm{d} U}{\mathrm{~d} z}\right|_{z \rightarrow \infty}=\left.\frac{\mathrm{d} W}{\mathrm{~d} z}\right|_{z \rightarrow \infty}=\left.\frac{\mathrm{d} C}{\mathrm{~d} z}\right|_{z \rightarrow \infty}=\left.\frac{\mathrm{d} P}{\mathrm{~d} z}\right|_{z \rightarrow \infty}=0 .
\end{aligned}
$$

These equations are discretized in the $z$-direction using Chebyshev collocation (Canuto et al. 2006). The collocation points $\xi_{j}=-\cos \left[(j-1) \pi /\left(N_{z}-1\right)\right]$, with $j=1, \ldots, N_{z}$, are mapped onto the interval $0 \leqslant z \leqslant z_{\max }$ with the transformation

$$
z_{j}=\frac{z_{h}\left(1+\xi_{j}\right)}{1-\xi_{j}+2 z_{h} / z_{\max }} .
$$

Approximately one half of the total number of collocation points are thus placed in the interval $0 \leqslant z \leqslant z_{h}$. Typically, $z_{h}=5, z_{\max }=200$ and $N_{z}=120$ are used, but it can be advantageous to increase $z_{h}$ and $z_{\max }$ for very small wavenumbers $\alpha$. The discretized equations (2.20)-(2.28) constitute a generalized eigenvalue problem $\boldsymbol{A} \boldsymbol{Q}=\sigma \boldsymbol{B} \boldsymbol{Q}$ (see the Appendix) that is solved using a QR algorithm, as implemented in the Lapack library. The real part $\sigma_{r}$ of the eigenvalue $\sigma=\sigma_{r}+\mathrm{i} \sigma_{i}$ is the temporal growth rate, the negative imaginary part $-\sigma_{i}$ is the angular frequency of perturbations with prescribed wavenumber $\alpha$. The associated phase velocity is obtained as $v_{p h}=-\sigma_{i} / \alpha$. The complex eigenvector $\boldsymbol{Q}$ contains all $z$-dependent perturbation amplitude functions.

\section{Flow over a non-evolving bed}

We will first investigate the structure of fluid perturbations in the boundary-layer flow over a non-evolving flat or wavy bottom. This assumption implies that the effect of deposition and erosion of sediment on the bottom topography is excluded throughout this section. The interface equation (2.5) is therefore replaced by (2.8), from which follows $\sigma E=0$. The linear system then admits one mode with $E \neq 0$ and $\sigma=0$, which represents a particular solution to the inhomogeneous boundaryvalue problem. This mode will be denoted as the 'wavy wall (WW) mode' in the following. All other solutions represent the eigenmodes of boundary-layer flow over a flat bottom $(E=0$ and $\sigma \neq 0)$.

In order to link our investigation to classical results of boundary-layer instability, we first consider in $\S 3.1$ a base flow with homogeneous density, $c_{0} \equiv 0$ and $C \equiv 0$. Buoyancy effects due to stratification are included in $\S 3.2$.

\subsection{Tollmien-Schlichting waves in the homogeneous boundary layer}

The temporal eigenvalue spectrum of a homogeneous boundary layer, for a given wavenumber $\alpha$, is known to be composed of a continuous branch (stable perturbations in the outer flow) and discrete eigenvalues (e.g. Mack 1976; Schmid \& Henningson 2001). The least stable mode of the discrete part of the spectrum is known as a 

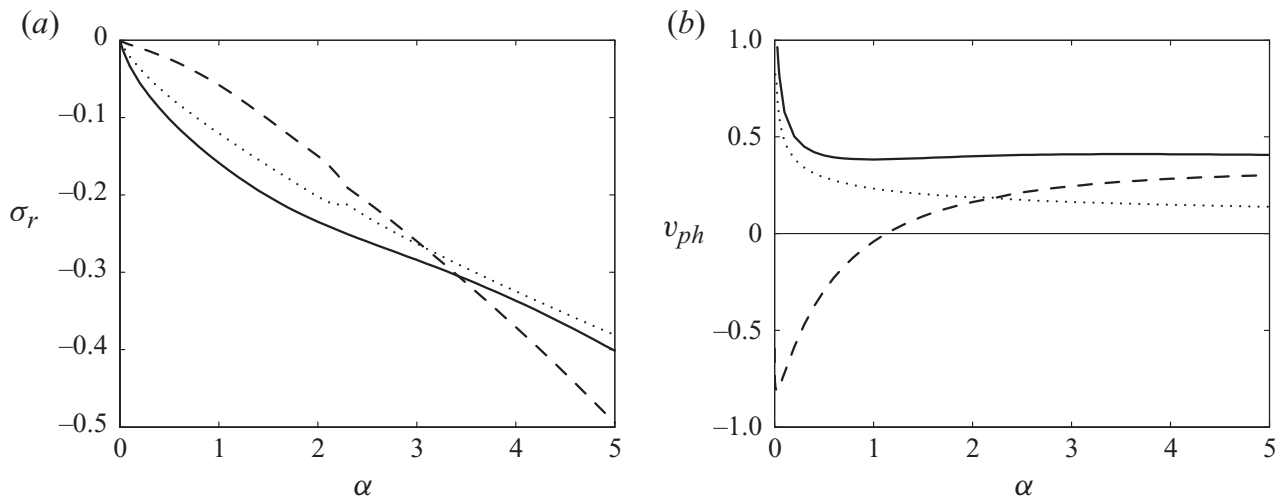

FIGURE 2. Eigenvalues of instability modes in the flow over a non-evolving bed: - , TS mode in homogeneous flow; $\cdots$, TS mode in stratified flow; --, internal wave mode in stratified flow. (a) Temporal growth rate $\sigma_{r}$ as a function of wavenumber. (b) Corresponding phase velocity $v_{p h}$.

Tollmien-Schlichting (TS) wave, which displays peak perturbation amplitudes inside the boundary layer and propagates at a phase velocity slower than the outer flow. The phase velocity and the growth rate of the TS mode, as obtained for the present velocity profile (2.17) with $c_{0} \equiv 0$, are shown in figure 2 (solid lines). In qualitative agreement with the results of Mack (1976) for the Blasius boundary layer, the phase velocity is approximately constant at 0.4 , except for very low wavenumbers, where it tends towards the outer flow velocity. Figure 2(a) shows that the TS mode is stable at all wavenumbers for the present parameters.

In the present case of a non-evolving wavy bottom, the WW mode represents the passive linear flow response to bottom perturbations with zero growth rate and zero phase velocity. The associated vertical distributions of fluid velocity and pressure perturbations $|W|$ and $|P|$, for $\alpha=1$, are displayed in figure $3(a, b)$ by solid lines, alongside the corresponding eigenfunction shapes of the flat-bottom TS mode (dashed lines). The two are strikingly similar. The most distinctive difference results from the bottom boundary conditions (inset in figure $3 a$ ): the flat-bottom TS mode obeys a Neumann condition $W^{\prime}(0)=0$, whereas $W^{\prime}(0)$ of the WW mode follows from $U(0)=-E u_{0}^{\prime}(0)$ and the continuity equation. The comparison in figure 3 suggests that the WW mode may be regarded as a TS-like flow perturbation that is forced by the wavy bottom geometry.

\subsection{Internal waves in the stratified boundary layer}

The stability of the stratified base flow (2.17)-(2.18) is investigated in this section, still for the case of a non-evolving bottom geometry, $\sigma E=0$. The concentration boundary condition (2.27), which reflects an erosive sediment flux, is applied in these calculations in order to be consistent with the later results of $\S 4$. The present model therefore includes the effects of sediment deposition and erosion on the concentration field, although the bottom geometry does not change as a consequence. This simplification can be seen as the limiting case of heavy particles with vanishing volume.

Figure 2 displays by dotted lines the phase velocity and the growth rate of the flat-bottom TS mode in the stratified setting. Both are somewhat affected by the stratification, but they still follow similar trends as in the homogeneous case. However, a new mode branch emerges in the stratified setting, represented by dashed lines in 

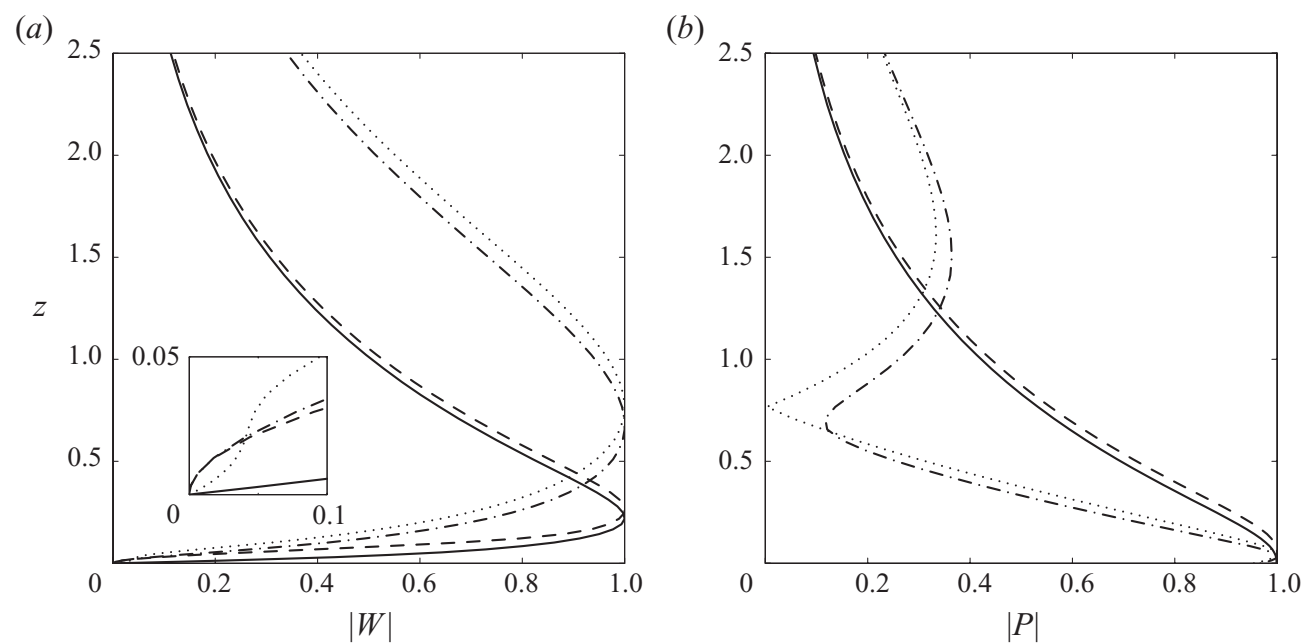

FIGURE 3. Perturbation eigenfunctions at wavenumber $\alpha=1$. WW mode (-) and TS mode $(--)$ in the homogeneous boundary layer; WW mode $(\cdots)$ and internal wave mode (-.-) in the stratified boundary layer. (a) Velocity perturbations $|W(z)|$; the inset displays a magnified view of the near-wall region. $(b)$ Pressure perturbations $|P(z)|$. All curves are normalized with respect to their individual maximum value.

figure $2(a, b)$. This mode corresponds to internal wave propagation in flow over a flat bottom, as will be demonstrated further below. The internal wave mode is stable for all wavenumbers (figure $2 a$ ); its phase velocity takes on negative values in the low-wavenumber regime $\alpha \lesssim 1$. The growth rates of the internal wave and TS modes are seen to approach each other around $\alpha=2.2$, where their phase velocities are equal. This behaviour is suggestive of a mixed instability character of both modes at higher wavenumbers, but this will not be further investigated here. Eigenfunctions of the internal wave mode are plotted by dash-dotted lines in figure $3(a, b)$. Note that the pressure functions feature vertical oscillations near the bottom. The growth rate and phase velocity of the WW mode are still zero. Its eigenfunctions in the stratified case, represented by dotted lines in figures $3(a)$ and $3(b)$, are now seen to closely follow those of the flat-bottom internal wave mode, as opposed to the TS mode (not plotted in figure 3).

Pressure perturbation eigenfunctions $|P(z)|$ of the WW mode are shown in figure 4(a) for three values of the wavenumber, $\alpha=0.1,1$ and 1.5. All three curves display oscillations near the bottom, and exponential decay without further oscillations beyond a critical distance. This distance may be taken as the position of the outermost maximum of the pressure eigenfunction (bullet symbols in figure $4 a$ ). All pressure eigenfunctions are normalized with respect to their maximum.

The transition from oscillatory to evanescent behaviour in figure $4(a)$ is characteristic for internal wave propagation in a medium with non-constant stratification. In a fluid at rest with constant density gradient $\rho^{\prime}=\mathrm{d} \rho / \mathrm{d} z$, internal waves may propagate into the outer flow if their frequency is not greater than the Brunt-Väisälä frequency, given in dimensional quantities as $\omega_{N}=\left(\rho^{\prime} g / \rho\right)^{1 / 2}$, where $g$ is the gravitational acceleration. At frequencies greater than $\omega_{N}$, internal waves are evanescent in such a flow (see for instance $\$ 2.2$ in Turner 1973). In the present configuration, the stratification varies in $z$, and the Brunt-Väisälä frequency must 
(a)

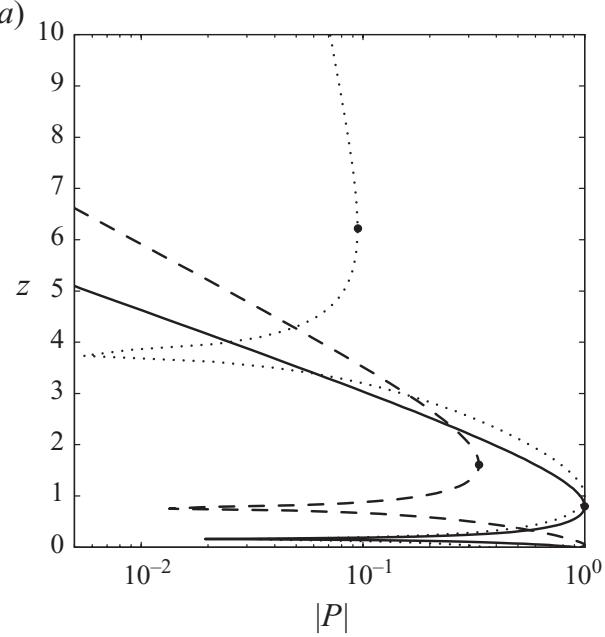

(b)

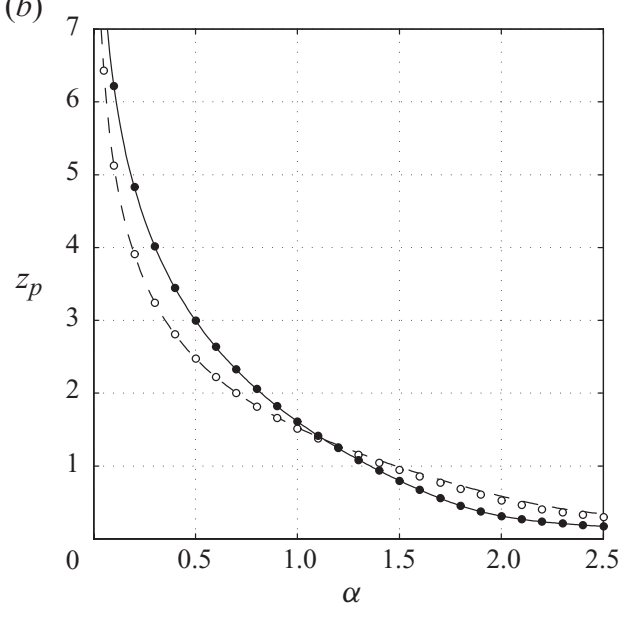

Figure 4. (a) Pressure eigenfunctions $|P(z)|$ of the WW mode at wavenumbers $\alpha=0.1(\cdots)$, $\alpha=1(--)$ and $\alpha=1.5(-)$. Symbols (๑) mark the position of the outermost pressure maximum. (b) Symbols: - , penetration distance obtained from the frequency criterion (3.2) at zero phase velocity; $\bullet$, position of the outermost pressure maximum of the wavy wall mode; --, penetration distance at the phase velocity $v_{p h}(\alpha)$ of the flat-bottom internal wave mode; $\circ$, outermost pressure maximum of the latter.

therefore be defined locally, in non-dimensional terms, as

$$
\omega_{N}(z)=\sqrt{-G \frac{\mathrm{d} c_{0}}{\mathrm{~d} z}(z)} .
$$

An eigenmode with wavelength $\alpha$ and phase velocity $v_{p h}$, relative to the bottom wall, has a Doppler-shifted frequency $\tilde{\omega}(z)=\alpha\left[u_{0}(z)-v_{p h}\right]$ in a reference frame moving with the local base flow velocity $u_{0}(z)$. At any vertical station $z$, the possibility of internal wave propagation at given values of $\alpha$ and $v_{p h}$ depends on the condition

$$
\alpha\left[u_{0}(z)-v_{p h}\right] \leqslant \omega_{N}(z) .
$$

With the family of base flow profiles defined in $\S 2.4$, a critical distance $z_{p}$ exists for each $\alpha$ of the WW mode $\left(v_{p h}=0\right)$, such that internal waves may propagate within a layer $0 \leqslant z \leqslant z_{p}$, but must be evanescent for $z>z_{p}$. This critical distance, characterized by $\alpha u_{0}\left(z_{p}\right)=\omega_{N}\left(z_{p}\right)$, will be denoted as the 'penetration distance' in the following.

The penetration distance $z_{p}$ as a function of $\alpha$, as predicted on the basis of the Brunt-Väisälä frequency, is plotted by a solid line in figure 4(b) for the WW mode in the standard base flow configuration. This analytical criterion is compared to the position of the outermost pressure perturbation maximum as found in the numerically computed eigenfunctions (filled circles). The agreement is exact within the limits of the spatial discretization. The dashed line and open circles in figure $4(b)$ represent the penetration distance and outermost pressure maximum of the flat-bottom internal wave mode $\left(v_{p h} \neq 0\right)$. In this case, excellent agreement is found at wavenumbers $\alpha \lesssim 1.5$. The small differences that occur at higher wavenumbers may be due to the influence of an added TS instability mechanism, as hinted in the discussion of figure $2(a)$.

Figure 4 clearly marks both the new mode branch (dashed lines in figure 2) and the WW mode in the stratified boundary layer as manifestations of internal wave 

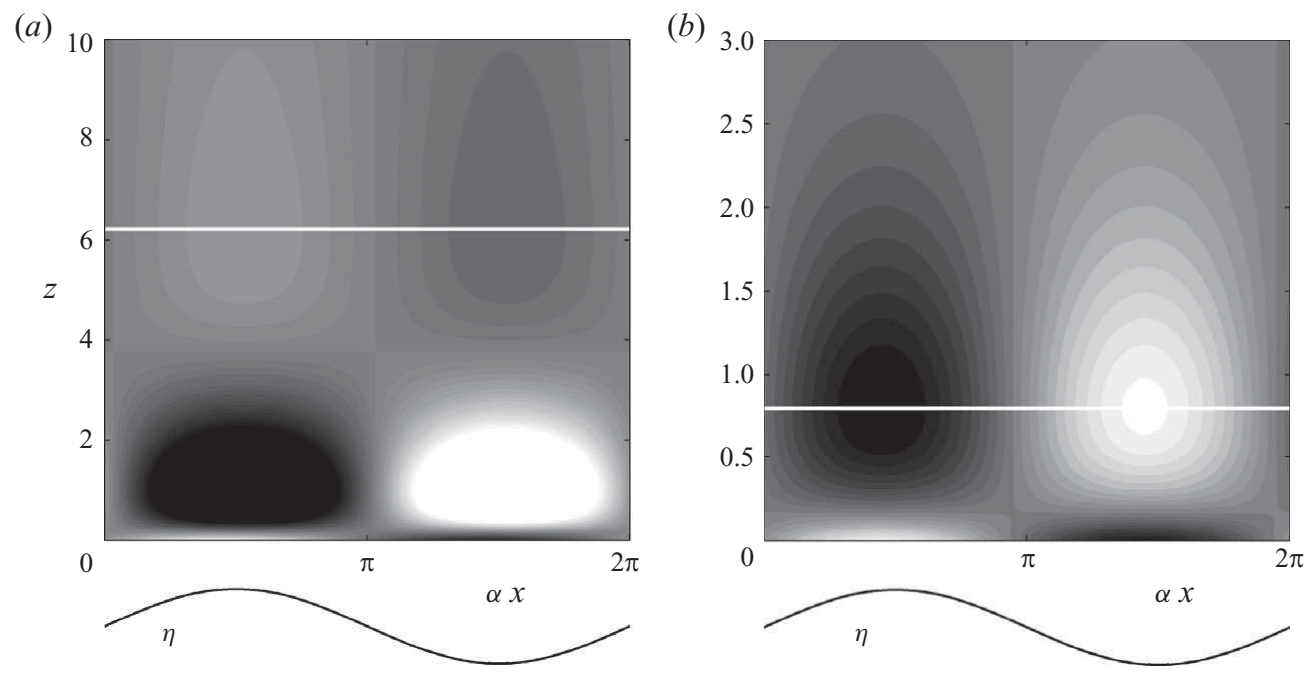

FIGURE 5. WW mode: two-dimensional pressure perturbation eigenfunctions $p^{\prime}(x, z)$ for (a) $\alpha=0.1$ and $(b) \alpha=1.5$. Light shades denote positive, dark shades negative values. White lines mark the penetration distance according to (3.2) for both cases. The lower curves $\eta=\sin \alpha x$ indicate the phase relation between the bottom wave and the fluid eigenmode.

propagation within a fluid layer near the bottom. The spatial shape of these internal waves is further examined in figure 5, which presents contour plots of the twodimensional pressure distributions of the wavy wall mode at $\alpha=0.1$ and $\alpha=1.5$. In both instances, as for any other value of $\alpha$, the pressure oscillations are found to form a checkerboard pattern, as is characteristic for standing waves in a wave duct. In analogy to acoustic waves inside a duct, for instance, such a pattern may result from the superposition of upward- and downward-propagating oblique waves that are reflected at the duct boundaries. Indeed, internal waves in a stratified medium propagate at oblique angles, and they undergo reflection at critical interfaces where further propagation is inhibited due to the Brunt-Väisälä frequency (Turner 1973). Note that the large penetration distance at $\alpha=0.1$ admits the presence of two pressure maxima along $z$ in the interior of the flow, whereas only one interior maximum is present at $\alpha=1.5$. The connection between the number of pressure maxima and the number of unstable wavenumber regimes will be further discussed in $\S 4.2$.

In conclusion, the analysis in this section has shown that wavy bottom perturbations in an unstratified boundary layer excite stationary Tollmien-Schlichting waves, whereas the flow response in the presence of stratification is dominated by internal wave dynamics.

\section{Flow over an evolving bed}

\subsection{Results for the standard set of parameters}

The bottom topography is now allowed to evolve in time, according to a two-way coupled interaction between the fluid perturbations and the sediment bed. The bed evolution is due to the competing effects of sedimentation and erosion, as described by (2.24), and its influence on the fluid perturbations arises from the time-dependent flow boundary conditions. In this coupled problem, the eigenvalue associated with the formerly stationary WW mode takes on finite values. The growth rate of this 


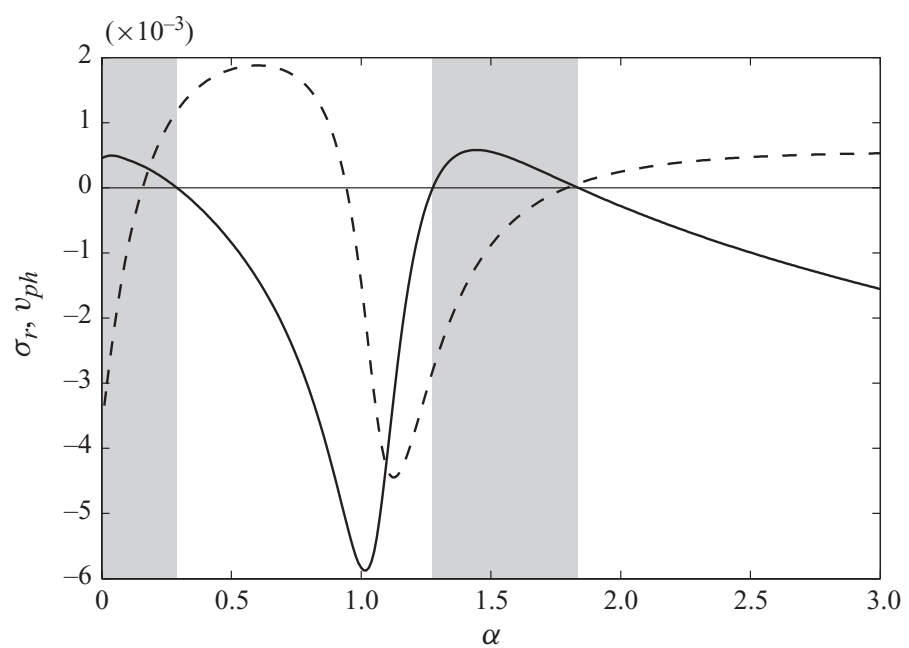

FiguRE 6. Temporal growth rate $\sigma_{r}(-)$ and phase velocity $v_{p h}(---)$ as functions of the wavenumber. Parameter values are $W_{s}=2 \times 10^{-4}, N=10^{-5}, G=10$ and $L=0.1$. Two unstable wavenumber regimes (shaded grey) exist for this set of parameters.

mode is plotted as a function of the wavenumber in figure 6 by a solid line, and the corresponding phase velocity is plotted by a dashed line. Instability is found over two separated wavenumber intervals $0 \leqslant \alpha \leqslant 0.29$ and $1.28 \leqslant \alpha \leqslant 1.84$ (grey shaded regions). At higher wavenumbers, the growth rate decreases monotonically with $\alpha$. The phase velocity is found to be negative over most of the unstable regimes, indicating a slow upstream migration of the instability wave.

As in the case of a non-evolving bottom wall (§3), all other eigenmodes are stable at all wavenumbers. Furthermore, the WW mode is the eigenmode that involves by far the most significant bed deformation. While the bottom wave amplitude $|E|$ associated with each mode of the coupled system is non-zero, the impact of flow perturbations on the sediment bed evolution can be quantified for each mode by scaling $|E|$ with the kinetic energy of the fluid velocity perturbation as

$$
|\tilde{E}|=|E| / \int_{0}^{\infty}|U(z)|^{2}+|W(z)|^{2} \mathrm{~d} z .
$$

At $\alpha=1$, a value $|\tilde{E}|=5.09$ is found for the WW mode, $|\tilde{E}|=0.24$ for the flat-bottom internal wave mode, and all other modes have $|\tilde{E}|<0.05$. Our further discussion will be restricted to the WW mode.

The non-zero value of the temporal eigenvalue $\sigma$ of the wavy wall mode is implicitly prescribed by the interface equation (2.24), which may be rewritten as

$$
\sigma=W_{s} c_{0}^{\prime}(0)-N u_{0}^{\prime \prime}(0)+\frac{W_{s}}{E} C(0)-\frac{N}{E} U^{\prime}(0) .
$$

Primes denote derivatives with respect to $z$. Substituting the base flow profiles (2.17)(2.18) into (4.2), the growth rate is found to be

$$
\sigma_{r}=\frac{N}{L}\left(\frac{1}{L}-1\right)+W_{s} \frac{|C(0)|}{|E|} \cos \Delta \phi_{C}-N \frac{\left|U^{\prime}(0)\right|}{|E|} \cos \Delta \phi_{U^{\prime}},
$$


where the complex eigenfunctions are taken in their polar form

$$
E=|E| \mathrm{e}^{\mathrm{i} \phi_{E}}, \quad C(0)=|C(0)| \mathrm{e}^{\mathrm{i} \phi_{C}}, \quad U^{\prime}(0)=\left|U^{\prime}(0)\right| \mathrm{e}^{\mathrm{i} \phi_{U^{\prime}}},
$$

and phase lags of the concentration and the shear variations at $z=0$, with respect to the bed elevation, are defined as

$$
\Delta \phi_{C}=\phi_{C}-\phi_{E}, \quad \Delta \phi_{U^{\prime}}=\phi_{U^{\prime}}-\phi_{E} .
$$

The three terms on the right-hand side of (4.3) reflect different mechanisms that influence the stability of the sediment bed evolution. The first term captures the effect of the base flow. The sign of this term, and thereby its stabilizing or destabilizing contribution, is determined by the parameter $L$ (the ratio of velocity to concentration boundary-layer thickness). The interpretation of the base flow term is fully analogous to the scenario described in Hall et al. (2008). Consider a flat sediment bed, where erosion and sedimentation due to the base flow are in balance. If $L<1$, the concentration boundary layer is thicker than the velocity boundary layer. An upward protrusion of the sediment bed then places the interface in a region where the base flow concentration, and consequently sediment deposition, decrease more slowly than the erosion by shear stress. The growth of the upward perturbation will therefore be reinforced. By the same reasoning, a downward perturbation of the interface will see the shear stress, and hence erosion, increase faster than concentration and sedimentation, causing the valley to deepen further. In the opposite case $L>1$, the velocity boundary layer is thicker than the concentration boundary layer. An upward perturbation will then see the concentration decrease more rapidly than the shear stress, so that erosion dominates on crests, and the perturbation decays.

The second term in (4.3) accounts for changes in the sediment deposition due to concentration perturbations at the bottom boundary. If the latter are in phase with the interface perturbation, $\cos \Delta \phi_{C}=1$, then deposition is increased on hills and decreased in troughs, and unstable growth is promoted. The third term in (4.3) represents changes of sediment erosion due to wall shear stress perturbations. This term has a maximum destabilizing effect if the wall shear stress is perfectly out of phase with the interface wave, $\cos \Delta \phi_{U^{\prime}}=-1$. Erosion in this case is stronger in troughs and weaker on crests. Note that both the concentration and the shear stress perturbations at $z=0$ are governed by the boundary conditions (2.25) and (2.27). These ensure that the total wall-tangential velocity is zero at the interface position $z=\eta$ and that the total concentration gradient corresponds to the erosive flux.

The phase velocity of an eigenmode is obtained from (4.2) as

$$
v_{p h}=-\frac{\sigma_{i}}{\alpha}=-\frac{W_{s}}{\alpha} \frac{|C(0)|}{|E|} \sin \Delta \phi_{C}+\frac{N}{\alpha} \frac{\left|U^{\prime}(0)\right|}{|E|} \sin \Delta \phi_{U^{\prime}}
$$

Sedimentation promotes a positive phase velocity for $-\pi<\Delta \phi_{C}<0$, when concentration perturbations take their maximum downstream of an interface crest. The enhanced deposition on the downslope flank then results in an apparent downstream migration of the bottom wave. Concentration perturbations cause a negative phase velocity (upstream migration) for $0<\Delta \phi_{C}<\pi$, when sedimentation is enhanced on the upslope flank. Conversely, erosion contributes to a positive phase velocity for $0<\Delta \phi_{U^{\prime}}<\pi$, and to a negative phase velocity for $-\pi<\Delta \phi_{U^{\prime}}<0$.

The influence of concentration and shear perturbations on the growth rate and phase velocity is illustrated in figure 7. The dotted and dashed lines display $C$ and $U^{\prime}$ at $z=0$ for a situation with phase shifts $\Delta \phi_{C}=\pi / 2$ and $\Delta \phi_{U^{\prime}}=-\pi / 3$. If the flow direction is from left to right, the concentration perturbation in this example leads 


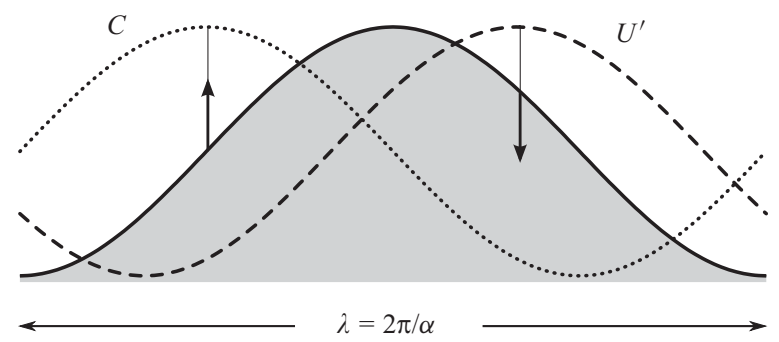

FIGURE 7. Schematic illustration of the effects of bottom concentration perturbation (dotted line) and bottom shear perturbation (dashed line) on the temporal evolution of a bottom interface wave (solid line). The phase shifts are chosen as $\Delta \phi_{C}=\pi / 2$ and $\Delta \phi_{U^{\prime}}=-\pi / 3$; arrows show the interface motion due to the individual contributions at their respective maximum locations. Both $C$ and $U^{\prime}$ cause the bottom wave to migrate towards the left. $C$ in this example does not contribute to the growth rate, $U^{\prime}$ has a stabilizing effect.
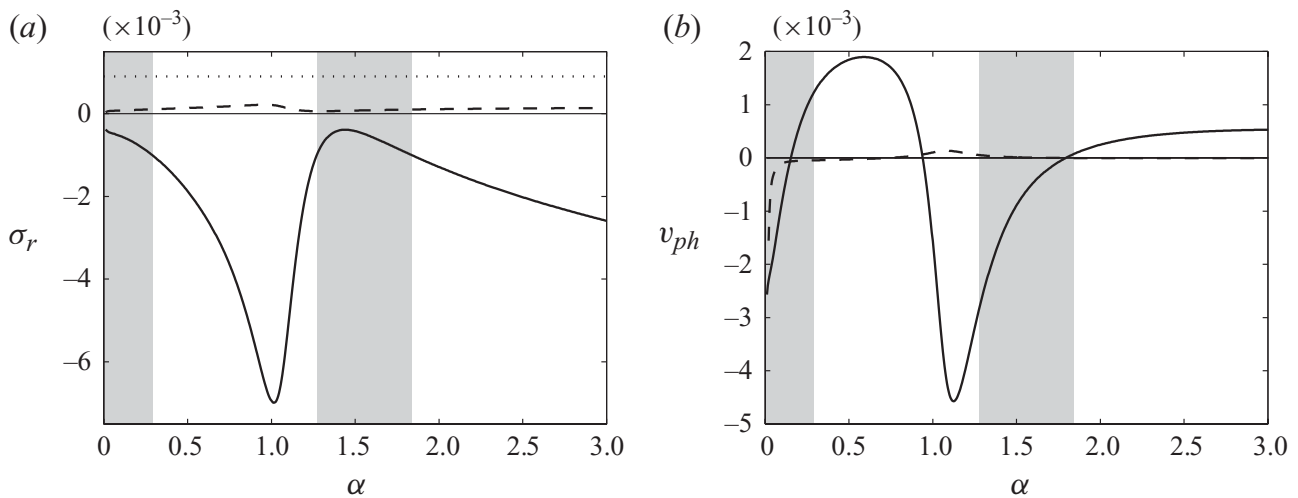

FIGURE 8. Individual contributions of the right-hand-side terms in (4.3) and (4.6). (a) Growth rate: - , shear perturbation term; ---, concentration perturbation term; $\cdots$, base flow term. (b) Phase velocity: - , shear perturbation term; ---, concentration perturbation term. Parameter values are the same as in figure 6 and unstable wavenumber intervals are shaded grey.

to a preferred deposition on the upslope flank, causing the interface there to rise as indicated by an arrow, while the shear perturbation leads to preferred erosion on the downslope flank, causing the interface to recede. As a result, the bottom wave will migrate upstream. With the chosen phase shifts, the concentration perturbation does not affect the bottom wave amplitude, and hence does not contribute to temporal growth or decay, whereas the shear perturbation induces a temporal decay. The base flow contribution is not represented in figure 7 .

Individual contributions to the growth rate and the phase velocity, as a function of $\alpha$ according to (4.3) and (4.6), for the same standard base flow configuration as in figure 6 are shown in figures $8(a)$ and $8(b)$. The base flow contribution to the growth rate, represented by a dotted line in figure 8 , does not depend on $\alpha$. Since $L=0.1<1$, the base flow effect is destabilizing. Perturbations of the sediment deposition, caused by $C(0)$ and plotted by a dashed line, also add to the instability at all wavenumbers, although the magnitude of their contribution is negligible at the present parameter setting. Variations of the total growth rate in $\alpha$ are almost exclusively due to erosion associated with shear perturbations, shown by a solid line. Similarly, shear perturbations dominate the variations of the phase velocity, although 


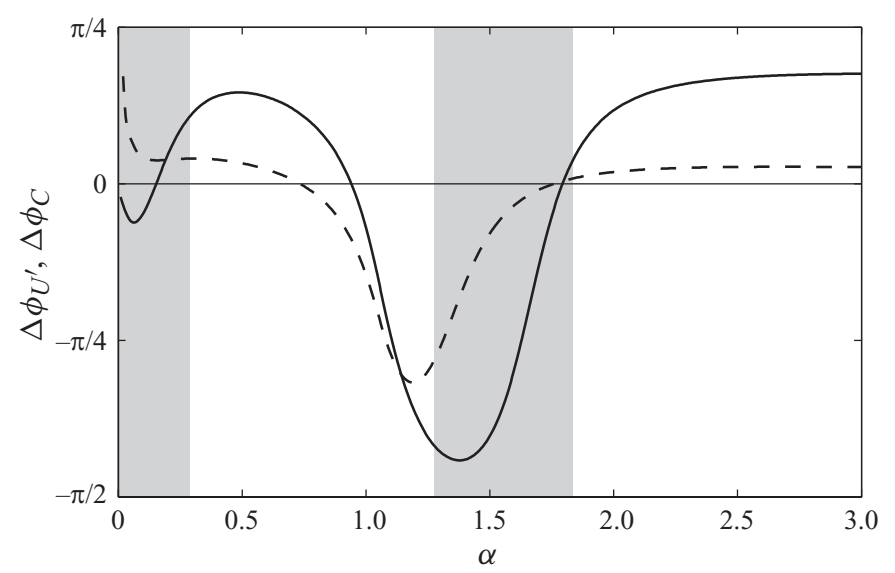

FIGURE 9. Phase lags: - $\Delta \phi_{U^{\prime}} ;---, \Delta \phi_{C}$. Unstable wavenumber intervals are shaded grey.

perturbational sediment deposition contributes to the negative phase velocity at very low wavenumbers $\alpha<0.1$.

It may seem surprising that instability is found in the zero-wavenumber limit. The analysis in $\S 3$ of flow over a non-evolving bed showed no instability of fluid perturbations at $\alpha=0$. In the case of an evolving bed, a zero-wavenumber perturbation of $\eta$ can be interpreted as a perturbation of the average interface velocity $w_{a v}$ (see $\S 2.3$ ). It is stressed again that the growth rate of the WW mode is entirely governed by the bed evolution model, according to (4.3). Whether an infinitesimal perturbation of the mean interface position $(\alpha=0)$ in the present context is stable, depends on whether the resulting perturbations of wall shear and sediment concentration are sufficiently stabilizing to counter the wavenumber-independent base flow effect.

The phase lags $\Delta \phi_{U^{\prime}}$ and $\Delta \phi_{C}$ of shear and concentration perturbations at $z=0$ are plotted in figure 9. Both quantities present variations and multiple changes of sign in the low-wavenumber regime $\alpha \lesssim 2$, but they approach constant positive values at higher wavenumbers. Fluid perturbations at low $\alpha$ near the bottom have been found to be dominated by internal wave propagation (see figures 4 and 5), whereas the penetration depth of internal waves nearly vanishes at $\alpha>2$. It appears that instability and upstream migration in the present framework relies on the presence of internal wave propagation. This connection has also been surmised by Flood (1988), albeit in the context of sediment waves formed by bottom currents with constant stratification. For such a setting, Flood (1988) advanced a qualitative explanation for upstream migration, based on streamline patterns that correspond to upwardpropagating internal waves. In the setting considered in the present paper, the internal wave patterns that govern erosional and depositional perturbations are more complex, owing to the presence of downward-propagating reflected waves. The effect of internal wave generation on the phase lags $\Delta \phi_{U^{\prime}}, \Delta \phi_{C}$, and thereby on the growth rate and phase velocity of bottom perturbations, cannot be inferred a priori from a qualitative argument.

\subsection{Influence of stratification}

The results in $\S 4.1$ have demonstrated that the flow/bed interaction through erosion and deposition perturbations results from the internal wave patterns that form in a wave-duct layer at the bottom. The existence of these internal waves in the fluid depends on the presence of a negative base flow density gradient $\mathrm{d} \rho_{0} / \mathrm{d} z<0$. In 

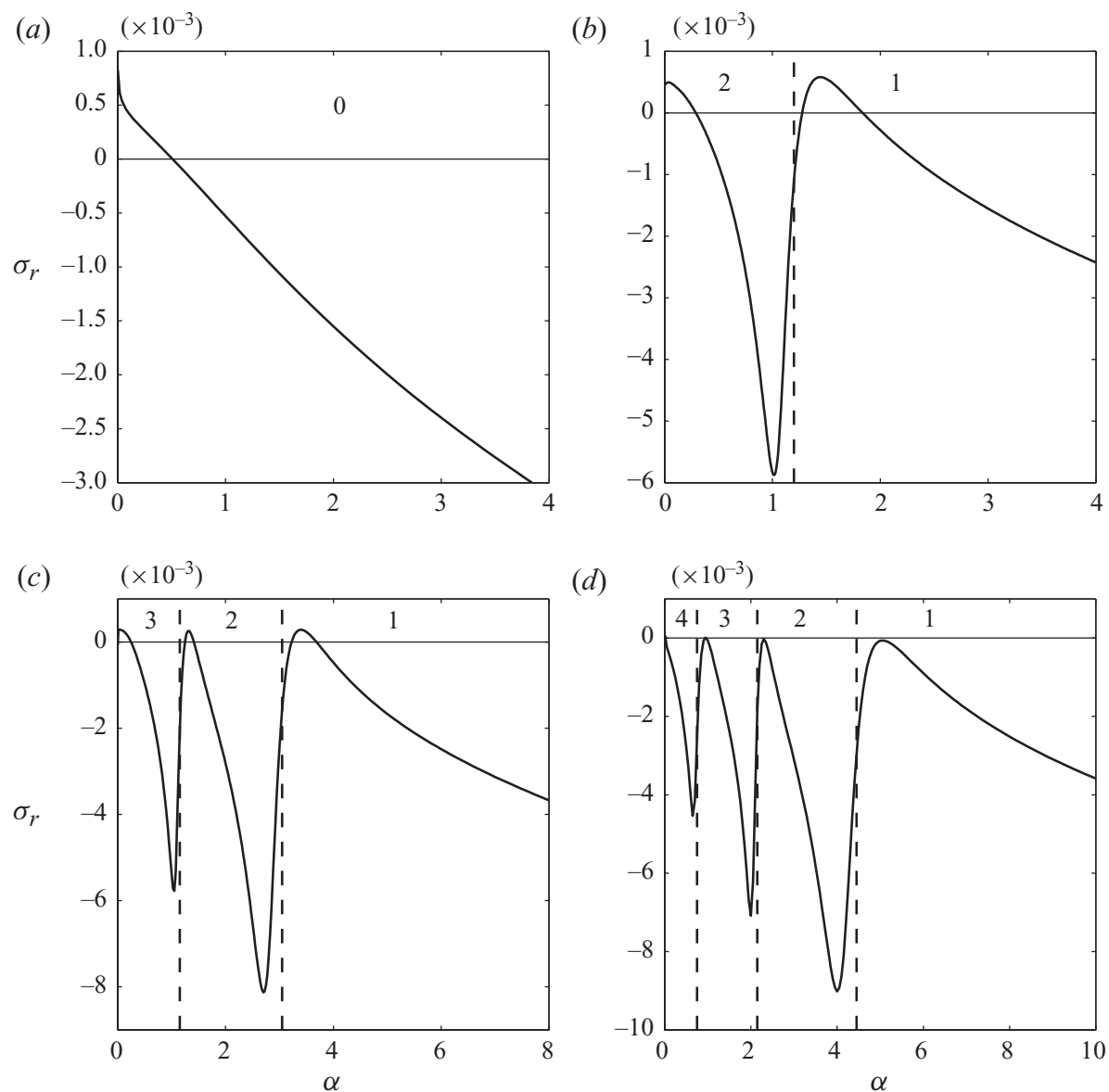

FIGURE 10. Growth rates of the instability mode at various values of the gravity parameter. (a) $G=0 ;(b) G=10 ;(c) G=30$ and $(d) G=50$. Dashed lines separate various wavenumber regimes, each one characterized by a number of pressure maxima in the interior of the fluid, as indicated in the diagrams.

this section, it will be investigated how the magnitude and vertical variation of the density gradient affects the growth rate and the modal shape of the coupled flow/bed instability. The density gradient follows from the base flow concentration profile (2.18) and from the reduced gravity $g^{\prime}$, which is contained in the definition of the gravity parameter $G$ (see $\S 2.3$ ), such that

$$
\frac{\mathrm{d} \rho_{0}}{\mathrm{~d} z} \propto G \frac{\mathrm{d} c_{0}}{\mathrm{~d} z} \propto \frac{G N}{L W_{s}} .
$$

The parameters that govern the concentration profile, $N, L$ and $W_{s}$, affect the dispersion relation in several ways, whereas the influence of $G$ is isolated in the buoyancy term of the $z$-momentum equation (2.22), which provides the link between fluid stratification and flow dynamics. The effect of $G$ on the flow/bed interaction is therefore considered first.

In the limiting case $G=0$, the sediment concentration causes no buoyancy effects, and internal waves cannot form. With all other parameters kept at the same values as before, the growth rate for this configuration is displayed in figure 10(a). Instability 
(a)

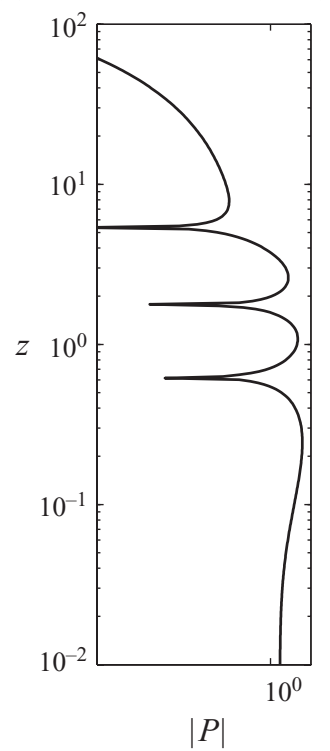

(b)

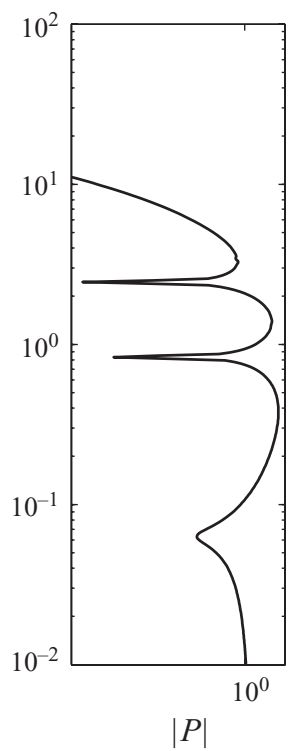

(c)

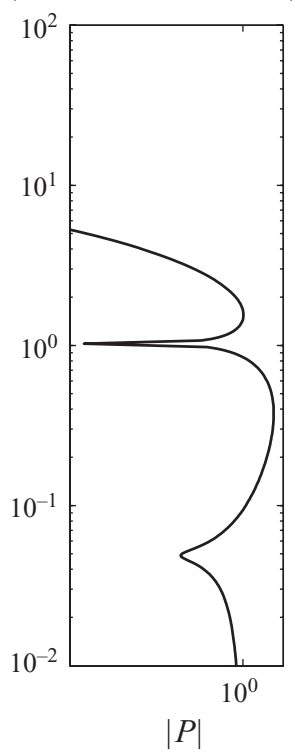

(d)

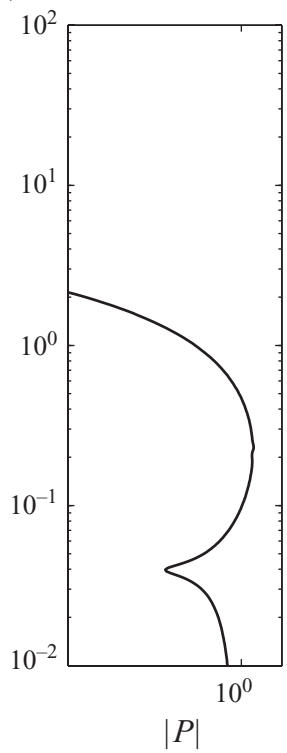

FIGURE 11. Pressure eigenfunctions from different wavenumber regimes, as marked in figure $10(d)$ for $G=50$. (a) $\alpha=0 ;(b) \alpha=0.95 ;(c) \alpha=2.3$ and $(d) \alpha=5.05$.

is still found at very low wavenumbers, but the growth rate decreases monotonically with $\alpha$. All perturbation eigenfunctions in this case are found to decay exponentially in $z$ for all $\alpha$, similar to the unstratified setting of $\S 3.1$.

For comparison, the growth rate at the standard value $G=10$ is displayed again in figure $10(b)$. A dashed line separates two wavenumber regimes: for $\alpha \leqslant 1.23$ the pressure eigenfunction displays two maxima along $z$ in the interior of the fluid, whereas only one maximum is found for $\alpha>1.23$ (compare to figure $4 a$ ). Figures $10(c)$ and $10(d)$ show the growth rate variations at $G=30$ and 50 , respectively. Dashed lines again separate wavenumber intervals over which the pressure eigenfunction is characterized by a given number of maxima within the fluid. For all values of $G$ and $\alpha$, the two-dimensional pressure perturbations form checkerboard patterns similar to those of figure 5. At very low $\alpha$, the internal wave structure displays three vertical cells for $G=30$, and four cells for $G=50$. Within each wavenumber interval, the growth rates in figure $10(b-d)$ are seen to undergo similar cycles of increase and decrease.

Pressure eigenfunctions from the different wavenumber regimes at $G=50$ are displayed in figure 11. The four values of $\alpha$ correspond to the local maxima of the growth rate in figure $10(d)$. As the height of the wave-duct layer decreases continuously with higher wavenumbers, fewer vertical cells are sustained. The outermost pressure maximum is always located at the critical height $z_{c}$, as demonstrated for $G=10$ in figure 4 , whereas the bottom boundary condition is governed by the complex interaction of shear and sediment concentration. As the wavenumber is varied from one value represented in figure 11 to the next, the lowest pressure maximum continuously 'disappears' through the bottom boundary. Consequently, similar variations in the bottom shear and concentration perturbations occur over each such wavenumber regime. 


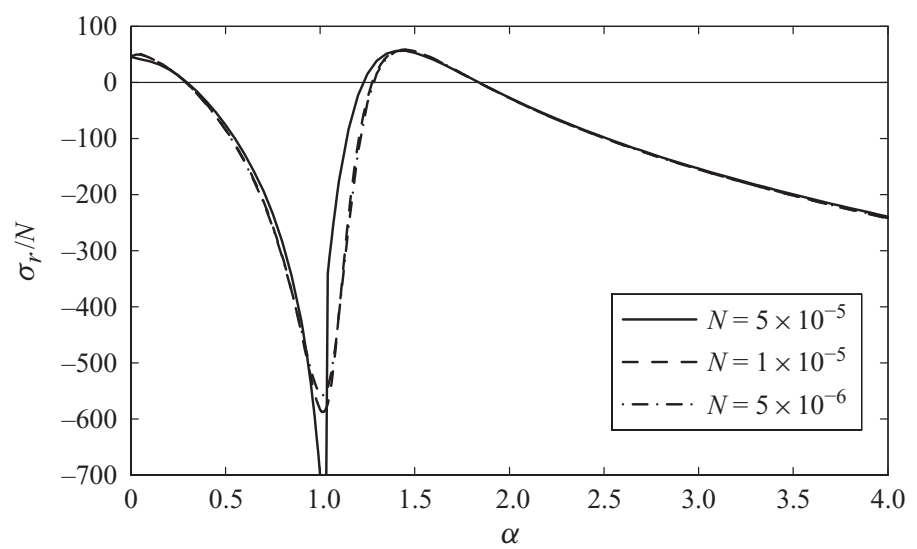

FIGURE 12. Scaled growth rate $\sigma_{r} / N$ for different values of the erosion parameter $N$. The product $G N$ is kept constant at a value of $10^{-4}$.

\subsection{Influence of parameters in the flow/bed interaction model}

The effect of flow perturbations on the bed evolution, as expressed by the temporal eigenvalue (4.3) and (4.6), is governed by the parameters $N, W_{s}$ and $L$. At the same time, these parameters influence the base flow stratification (4.7), and therefore the internal wave pattern near the bottom. The effect of the latter on the bed evolution has been described in $\S 4.2$. In this section, the influence of $N, W_{s}$ and $L$ on the growth rate via the flow/bed interaction model will be documented. In order to isolate these effects, the parameters will be varied individually, while at the same time their impact on the density gradient (4.7) will be compensated by adjusting the gravity parameter $G$.

The erosion parameter $N$ is considered first. Growth rates obtained for values of $N$ between $5 \times 10^{-6}$ and $5 \times 10^{-5}$ are shown in figure 12 ; each curve is scaled with its respective value of $N$. The gravity parameter is varied accordingly, such that $G N=10^{-4}$ in all cases. Since the dominant base flow and erosion contributions to the growth rate, according to (4.3), are linear in $N$, it is not surprising that the scaled curves in figure 12 approximately collapse.

The influence of the settling velocity $W_{s}$ on the instability is examined in figure 13 . The growth rate as a function of wavenumber is presented for values of $W_{s}$ that span two orders of magnitude. $G$ is varied to maintain a ratio of $G / W_{s}=5 \times 10^{4}$. As $W_{s}$ takes on smaller values, the two unstable wavenumber regimes are found to persist, but the unstable intervals become narrower, and the maximum growth rate continuously shifts to larger values of $k$. The decay in the stable high-wavenumber regime is stronger for small settling velocities.

As the ratio $L$ of the velocity over the concentration boundary layer thickness is varied, a constant ratio $G / L=100$ is maintained, and this ensures an unchanging base flow density gradient. However, the effect of the changed base flow velocity profile on the internal wave structure cannot be compensated. Furthermore, in the medium to high-wavenumber regime, the height of the wave duct increases as $L$ increases. Both of these effects cause changes in the growth rate variations $\sigma_{r}(\alpha)$, as evidenced in figure 14. Two trends are observed: first, the instability weakens as $L$ takes on larger values, and the flow is stable at $L \geqslant 0.3$. This is readily explained as the result of a weakened base flow contribution to the growth rate, given by the first term in (4.3). Second, with increasing $L$, the monotonic decay of the growth rate sets in at 


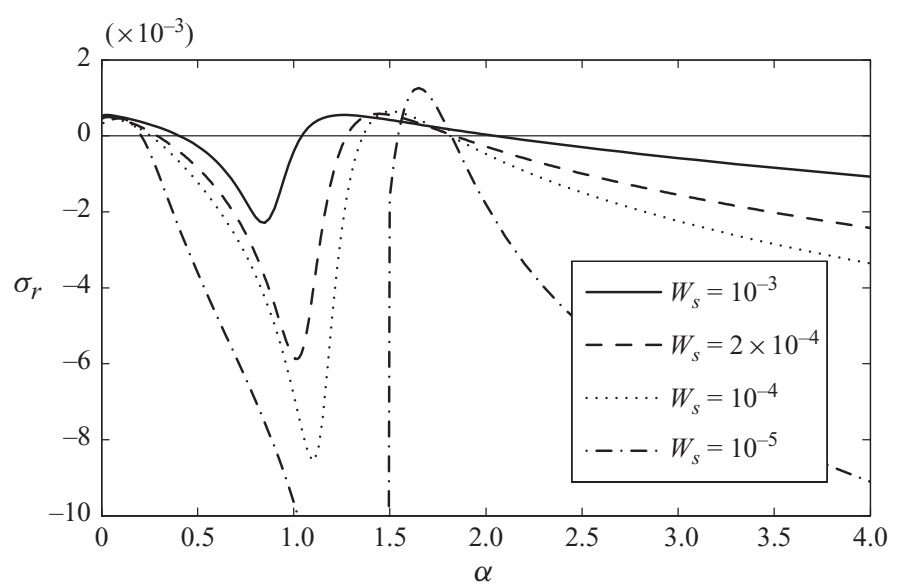

FIGURE 13. Growth rate for different values of the settling velocity $W_{s}$. The ratio $G / W_{s}$ is kept constant at a value of $5 \times 10^{4}$.

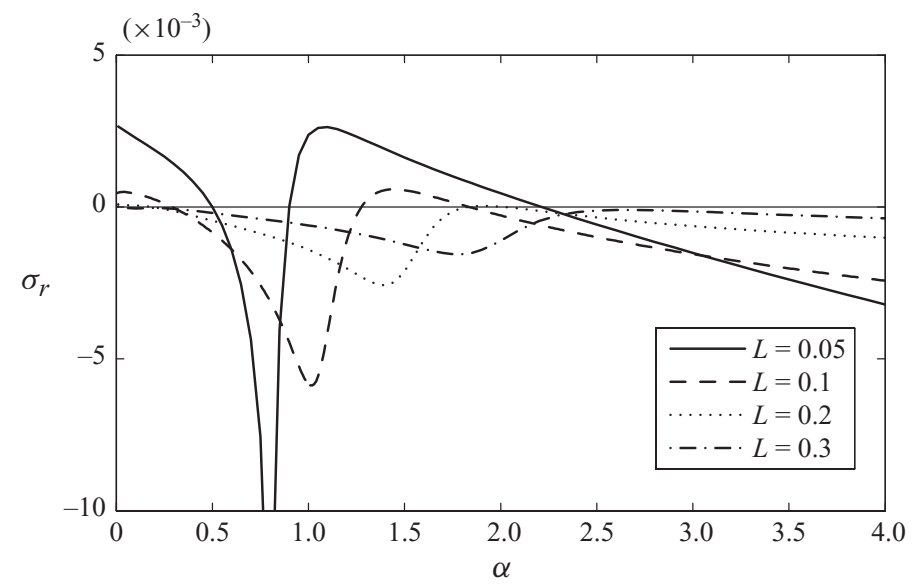

FIGURE 14. Growth rate for different values of the base flow parameter $L$. The ratio $G / L$ is kept constant at a value of 100 .

higher wavenumbers. This observation may be attributed to the increased penetration distance, which allows internal wave propagation over a larger wavenumber interval.

\section{Conclusions}

A temporal linear stability analysis of a stratified boundary layer flow over a wavy bottom has been presented, where the stratification was provided by suspended sediment. Flow parameters for the analysis were estimated such as to represent the boundary layer of a turbidity current, in order to relate the results to mechanisms of sediment wave formation. The choice of these parameters, as well as the dynamical model, follows the study of channel incision by Hall et al. (2008).

The flow over an unchanging bottom topography was considered first, and thus the one-way influence of bottom perturbations on the flow field was characterized. In an unstratified setting, flow perturbations caused by a wavy bottom are closely related to Tollmien-Schlichting waves. In a stratified setting, a wavy topography was seen 
to generate internal waves that propagate inside a fluid layer near the bottom. The stratified boundary layer therefore represents a wave duct for internal waves. The height of this layer could be determined analytically, in excellent agreement with the numerical results.

The analysis was then extended to account for the two-way coupled interaction between fluid and bed perturbations. In this setting, the flow induces slow changes of the bottom topography through deposition and erosion of sediment. Deposition was modelled as a downward convective flux of sediment, whereas erosion was treated as an upward diffusive flux. Small phase velocities and temporal growth rates of coupled perturbations were obtained from this interaction model; the bottom topography evolves on a slower scale than the characteristic time scale of the base flow. The fluid perturbations therefore were found to be nearly identical in the cases of unchanging and slowly changing bottom topography.

Growth rate and phase velocity of the fully coupled flow/bed perturbations follow directly from the interaction model. Instability was found at low wavenumbers near $\alpha=0$ for a variety of parameter settings. Depending on the base flow stratification, several separated unstable wavenumber ranges may exist. These have been linked to internal wave modes with different numbers of vertically aligned wave cells. At large wavenumbers, $\alpha>\mathcal{O}(1)$, perturbations were found to be stable for all investigated parameter combinations. The interface evolution equation allows one to explicitly identify three distinct contributions to the total growth rate, the first being associated with the balance of deposition and erosion in the base flow away from the interface, the second and third being associated with perturbations of deposition and erosion, respectively. The base flow contribution is destabilizing for $L<1$, i.e. when the velocity boundary layer is thinner than the sediment concentration layer, and it is independent of the wavenumber. The effect of depositional perturbations was found to be negligible over the considered range of parameters, whereas erosional perturbations may cause significant variations in the total growth rate as a function of the wavenumber. In the present context, erosional perturbations consistently had a stabilizing influence, such that the destabilizing effect of the base flow had to be sufficiently strong, requiring $L<0.3$, in order to yield a positive total growth rate. The importance of the base flow effect, as well as its physical interpretation, is fully consistent with the channel incision scenario described by Hall et al. (2008).

Conclusions about the relevance of the investigated instability problem for the formation of sediment waves must be drawn with caution. The present study characterizes the structure of internal waves that form inside a stratified boundary layer, in response to a wavy bottom topography. If the employed flow/bed interaction model adequately describes the essential physical processes, it has been demonstrated that these internal waves in turn may give rise to an unstable growth of bottom perturbations. Internal waves in the bottom boundary layer of a turbidity current therefore qualify as a possible mechanism for sediment wave formation. Unstable modes have been found to typically exhibit slightly negative phase velocities, which is consistent with the observation that sediment waves tend to slowly migrate upslope. For the standard set of flow parameters, the unstable low-wavenumber regime identified in $\S 4.1$ extends over the interval $0 \leqslant \alpha \leqslant 0.29$. Assuming that sediment waves typically have wavelengths between 1 and $10 \mathrm{~km}$, and that turbidity currents extend between 10 and $100 \mathrm{~m}$ in height, the non-dimensional wavenumbers fall in the range $\mathcal{O}\left(10^{-3}-10^{-1}\right)$. Despite all limitations of the present model, and the considerable uncertainty in the choice of appropriate parameters, the qualitative agreement with field observations is encouraging. 
Future investigations should extend the present formalism to flow configurations that include the upper region of a turbidity current. A distinct family of internal wave modes exists at the interface between the current body and the outer flow. The two-layer model study by Normark et al. (1980) presents plausible arguments that such waves may also be responsible for sediment wave formation. The influence of quasi-stationary internal waves, in various vertical regions of a turbidity current, on the bedform evolution remains to be investigated in a depth-resolved framework. Furthermore, the effect of bedload transport should be included in the flow/bed interaction model in future studies. Bedload transport is certainly crucial for the modelling of bottom currents, and its importance for sediment wave formation by turbidity currents cannot be excluded.

The assumption of constant eddy viscosity and diffusivity represents a strong simplification of the flow physics, and must be expected to significantly influence the quantitative results presented in this study. An accurate model for turbulence dynamics in turbidity currents, and for its effect on particle transport near an erodible bottom, is currently not available. A more faithful investigation of the effects of turbulence on the flow/bed interaction in the future may be based on turbulent mean profiles calculated from large-eddy simulations. Such investigations could also account for the influence of the streamwise mean flow development on the instability dynamics, which was excluded in the present analysis.

L.L. was partially supported by a Feodor-Lynen fellowship of the Alexander-vonHumboldt Foundation. The authors gratefully acknowledge financial support by BG Group via a grant to B.K. and L.L., and through NSF grant CBET-0854338.

\section{Appendix. The temporal eigenvalue problem}

The matrices of the eigenvalue problem $\boldsymbol{A} \boldsymbol{Q}=\sigma \boldsymbol{B} \boldsymbol{Q}$ defined in $\S 2.4$ follow from $(2.20)-(2.24)$ as

$$
\boldsymbol{A}=\left(\begin{array}{ccccc}
\mathrm{i} \alpha \mathbf{1} & \boldsymbol{d}_{z} & \mathbf{0} & \mathbf{0} & 0 \\
\boldsymbol{M}_{1} & -\boldsymbol{D}\left(u_{0}^{\prime}\right) & -\mathrm{i} \alpha \mathbf{1} & \mathbf{0} & 0 \\
\mathbf{0} & \boldsymbol{M}_{1} & -\boldsymbol{d}_{z} & -G \mathbf{1} & 0 \\
\mathbf{0} & -\boldsymbol{D}\left(c_{0}^{\prime}\right) & \mathbf{0} & \boldsymbol{M}_{2}+W_{s} \boldsymbol{d}_{z} & 0 \\
M_{3} & 0 & 0 & W_{s} & M_{4}
\end{array}\right),
$$

$$
B=\left(\begin{array}{lllll}
0 & 0 & 0 & 0 & 0 \\
1 & 0 & 0 & 0 & 0 \\
0 & 1 & 0 & 0 & 0 \\
0 & 0 & 0 & 1 & 0 \\
0 & 0 & 0 & 0 & 1
\end{array}\right)
$$

with the definitions

$$
\begin{aligned}
& \boldsymbol{M}_{1}=-\mathrm{i} \alpha \boldsymbol{D}\left(u_{0}\right)+\frac{1}{R e}\left(-\alpha^{2} \mathbf{1}+\boldsymbol{d}_{z z}\right), \\
& \boldsymbol{M}_{2}=-\mathrm{i} \alpha \boldsymbol{D}\left(u_{0}\right)+\frac{1}{P e}\left(-\alpha^{2} \mathbf{1}+\boldsymbol{d}_{z z}\right),
\end{aligned}
$$




$$
\begin{aligned}
& M_{3}=-\left.N \mathrm{~d}_{z}\right|_{z=0}, \\
& M_{4}=\left[W_{s} c_{0}^{\prime}-N u_{0}^{\prime \prime}\right]_{z=0} .
\end{aligned}
$$

First- and second-order derivative operators are written as $\boldsymbol{d}_{z}$ and $\boldsymbol{d}_{z z}$, primes denote derivatives of base flow quantities with respect to $z$, and $\boldsymbol{D}(\boldsymbol{a})$ denotes a matrix with the elements of vector $\boldsymbol{a}$ on the diagonal. The bottom boundary conditions (2.25)-(2.27) replace the first lines in matrices $\boldsymbol{A}$ and $\boldsymbol{B}$ in the blocks representing the momentum and concentration equations. The outer boundary conditions (2.28) replace the last lines in blocks representing the continuity, momentum and concentration equations.

\section{REFERENCES}

Allen, J. R. L. 1970 Physical Processes of Sedimentation. Unwin University Books.

Blanchette, F., Strauss, M., Meiburg, E., Kneller, B. \& Glinsky, M. E. 2005 High resolution numerical simulations of resuspending gravity currents: conditions for self-sustainment. $J$. Geophys. Res. C: Oceans 110 (C12022), doi:10.10129/2005JC002927.

Canuto, C., Hussaini, M. J., Quarteroni, A. \& Zang, T. A. 2006 Spectral Methods, Fundamentals in Single Domains. Springer.

Colombini, M. 2004 Revisiting the linear theory of sand dune formation. J. Fluid Mech. 502, 1-16.

Colombini, M. \& Stocchino, A. 2008 Finite-amplitude river dunes. J. Fluid Mech. 611, 283-306.

Engelund, R. \& Fredsoe, J. 1982 Sediment ripples and dunes. Annu. Rev. Fluid Mech. 14, 13-37.

Fildani, A., Normark, W. R., Kostic, S. \& Parker, G. 2006 Channel formation by flow-stripping: large-scale scour features along the Monterey East Channel and their relation to sediment waves. Sedimentology 53, 1265-1287.

Flood, R. D. 1988 A lee wave model for deep-sea mudwave activity. Deep-Sea Res. 35 (6), 973-983.

Garcia, M. H. \& Parker, G. 1993 Experiments on the entrainment of sediment into resuspension by a dense bottom current. J. Geophys. Res. 98, 4793-4807.

Hall, B., Meiburg, E. \& Kneller, B. 2008 Channel formation by turbidity currents: NavierStokes-based linear stability analysis. J. Fluid Mech. 615, 185-210.

Heezen, B. C., Tharp, M. \& Ewing, M. 1959 The floors of the oceans. 1. The North Atlantic. GSA Spec. Pap. 65, 122 pp.

Hill, P. S. 1998 Controls on floc size in the sea. Oceanography 11 (2), 13-18.

Kennedy, J. F. 1969 The formation of sediment ripples, dunes and antidunes. Annu. Rev. Fluid Mech. 1, 147-168.

Kubo, Y. \& NAKAJIMA, T. 2002 Laboratory experiments and numerical simulation of sediment-wave formation by turbidity currents. Mar. Geol. 192, 105-121.

Lewis, K. B. \& PAntin, H. M. 2002 Channel-axis, overbank and drift sediment waves in the southern Hikurangi Trough, New Zealand. Mar. Geol. 192, 123-151.

Lonsdale, P. F. \& Hollister, C. D. 1979 A near-bottom traverse of Rockall Trough: hydrographic and geological inferences. Oceanol. Acta 2, 91-105.

MACK, L. M. 1976 A numerical study of the temporal eigenvalue spectrum of the Blasius boundary layer. J. Fluid Mech. 73, 497-520.

Meiburg, E. \& Kneller, B. 2010 Turbidity currents and their deposits. Annu. Rev. Fluid Mech. 42, $135-156$.

Migeon, S., Savoye, B., Zanella, E., Mulder, T., Faugéres, J.-C. \& Weber, O. 2001 Detailed seismic-reflection and sedimentary study of turbidite sediment waves on the Var Sedimentary Ridge (SE France): significance for sediment transport and deposition and for the mechanisms of sediment wave construction. Mar. Petrol. Geol. 18, 179-208.

NAKajima, T. \& Satoh, M. 2001 The formation of large mudwaves by turbidity currents on the levees of the Toyama deep-sea channel, Japan Sea. Sedimentology 48, 435-463.

Necker, F., Härtel, C., Kleiser, L. \& Meiburg, E. 2002 High-resolution simulations of particledriven gravity currents. Intl J. Multiphase Flow 28, 279-300.

Necker, F., Härtel, C., Kleiser, L. \& Meiburg, E. 2005 Mixing and dissipation in particle-driven gravity currents. J. Fluid Mech. 545, 339-372. 
Normark, W. R., Hess, G. R., Stow, D. A. V. \& Bowen, A. J. 1980 Sediment waves on the Monterey fan levee: a preliminary physical interpretation. Mar. Geol. 37, 1-18.

Normark, W. R., Piper, D. J. W., Posamentier, H., Pirmez, C. \& Migeon, S. 2002 Variability in form and growth of sediment waves on turbidite channel levees. Mar. Geol. 192, 23-58.

PARKer, G. 1978 Self-formed straight rivers with equilibrium banks and mobile bed. Part 1. The sand-silt river. J. Fluid Mech. 89 (1), 109-125.

Peakall, J., McCaffrey, W. D. \& Kneller, B. C. 2000 A process model for the evolution, morphology, and architecture of sinuous submarine channels. J. Sedim. Res. 70 (3), 434-448.

Piper, D. J. W. \& Normark, W. R. 1983 Turbidite depositional patterns and flow characteristics, Navy submarine fan, California Borderland. Sedimentology 30, 681-694.

Queney, P. 1948 The problem of air flow over mountains: a summary of theoretical studies. Bull. Am. Meteorol. Soc. 29, 16-26.

Raudkivi, A. J. 1966 Bed forms in alluvial channels. J. Fluid Mech. 26 (3), 507-514.

REYNOLDS, A. J. 1976 A decades' investigation of the stability of erodible stream beds. Nord. Hydrol. 7, 161-180.

Savoye, B., Piper, D. J. W. \& Droz, L. 1993 Plio-Pleistocene evolution of the Var deep-sea fan off the French Riviera. Mar. Petrol. Geol. 10, 550-571.

Schmid, P. J. \& Henningson, D. S. 2001 Stability and Transition in Shear Flows. Springer.

Shanmugam, G. 2006 Deep-water Processes and Facies Models: Implications for Sandstone Petroleum Reservoirs, p. 86. Elsevier.

Stacey, M. W. \& Bowen, A. J. 1988 The vertical structure of density and turbidity currents: Theory and observations. J. Geophys. Res. 93, 3528-3542.

Sun, T. \& PARKer, G. 2005 Transportational cyclic steps created by flow over an erodible bed. Part 2. Theory and numerical simulation. J. Hydraul. Res. 43 (5), 502-514.

TAKI, K. \& Parker, G. 2005 Transportational cyclic steps created by flow over an erodible bed. Part 1. Experiments. J. Hydraul. Res. 43 (5), 488-501.

Turner, J. S. 1973 Buoyancy Effects in Fluids. Cambridge University Press.

Wynn, R. B., Masson, D. G., Stow, D. A. V. \& Weaver, P. P. E. $2000 a$ Turbidity current sediment waves on the submarine slopes of the western Canary Islands. Mar. Geol. 163, 185-198.

Wynn, R. B. \& Stow, D. A. V. 2002 Classification and characterisation of deep-water sediment waves. Mar. Geol. 192, 7-22.

Wynn, R. B., Weaver, P. P. E., Ercilla, G., Stow, D. A. V. \& Masson, D. G. $2000 b$ Sedimentary processes in the Selvage sediment-wave field, NE Atlantic: new insights into the formation of sediment waves by turbidity currents. Sedimentology 47, 1181-1197. 